- ihr ist ein Quellenverzeichnis beizulegen und eine Bestätigung, dass das Urheberrecht beachtet wurde;

- zum Wettbewerb können Arbeiten von Einzelnen oder von Gruppen (bis zu fünf Mitarbeitern) eingereicht werden;

- die Arbeiten dürfen nicht älter als zwei Jahre sein;

- den Arbeiten ist eine Kurzbeschreibung (max. 2 Seiten DIN A 4) beizufügen, die Auskunft über Zweck, Zielgruppe und Intention, Wahl der Darstellungsmittel und Herstellungsverfahren gibt. Bei rechnergestützt hergestellten Arbeiten sind die technischen Voraussetzungen anzugeben;

- die Arbeiten müssen auf der Rückseite mit Namen, Anschrift und E-Mail-Adresse der Teilnehmer sowie der Ausbildungsstätte oder der Hochschule versehen sein;

- den Arbeiten ist ein adressierter Aufkleber für die Rücksendung beizulegen.

Mit der Teilnahme am Wettbewerb werden die Wettbewerbsregeln anerkannt. Dazu gehört gem. § 12 UrhG auch die Erklärung der Preisträger, als Inhaber des Veröffentlichungsrechts an ihren kartographischen Arbeiten deren öffentliche Präsentation der Kartographie-Stiftung Ravenstein unwiderruflich zu gestatten.

Zum Jahresende 2014 schieden der frühere Präsident des BKG, Herr Prof. Dr. Dietmar Grünreich, als langjähriges Mitglied des Vorstands und Herr Referatsleiter Dipl.-

Geograph Guido Bodingbauer, Juror und Vorprüfer, aus der Stiftung aus. Die Nachfolger - Herr Prof. Dr.-Ing. Hansjörg Kutterer, Präs. des BKG, als Mitglied des Vorstands (Abwesenheitsvertreter: AL GI Dr. Bernd Richter) und Dr. Michael Hovenbitzer, RL GI 7 als Mitglied der Jury und Leiter der Vorprüfung - unterstützen seit Anfang 2015 die Arbeit der "KartographieStiftung Ravenstein". Damit ist die unverzichtbare Hilfe des Bundesamtes für Kartographie und Geodäsie weiterhin gesichert.
Die genauen Termine für die Abgabe der Beiträge im Sommer 2015 werden in den $\mathrm{KN}, \mathrm{H}$. 2/2015 veröffentlicht.

Weitere Auskünfte über den Wettbewerb gibt es bei dem Vorsitzende der Jury, Herr Dipl.-Geograph Dirk Zellmer, Berlin, unter der E-Mail: DirkZellmer@web.de sowie auf der Website der Ausbildungskommission der DGfK http://geomatik-ausbildung de wo auch die Preisträger der Vorjahre dargestellt werden.

Dr. Horst Schöttler

Vorstandsvorsitzender der Kartographie-Stiftung Ravenstein

Dirk Zellmer

Vorsitzender der Jury

\section{Neujahrsempfang der DGfK in Berlin}

„Die Vereinten Nationen haben das internationale Jahr der Karte ausgerufen, um auf die Bedeutung von Karten in unserer Gesellschaft aufmerksam zu machen..." gab Prof. Dr. Georg Gartner, der Präsident der ICA in einem Grußwort auf dem Neujahrsempfang der DGfK bekannt. Unter der Beteiligung von knapp 100 Personen gab unsere Gesellschaft, vertreten durch den Präsidenten Prof. Dr.-Ing. Manfred Weisensee diesen Empfang am 15.01.2015 in Berlin. Die Veranstaltung wurde gemeinsam ausgerichtet von der Sektion Berlin-Brandenburg, unter der Leitung von Dipl.-Ing. Horst Kremers und der DGfK-Bund. Als Veranstaltungsort stand der ideal geeignete SimónBolivar-Saal der Staatsbibliothek zu Berlin zur Verfügung. Die Ansprache von Georg Gartner war mitreißend und stimmte das Auditorium auf zahlreiche Aktivitäten im Jahr der Karte 2015 und 2016 unter dem Motto „We love Maps" ein. Er erläuterte die Intension der Vereinten Nationen, mit diesen Aktivitäten die hohe Bedeutung von Karten in der Gesellschaft heute und in der Zukunft zu unterstreichen. Der Festvortrag wurde von dem Geoinformatiker Prof. Dr. Manfred Ehlers

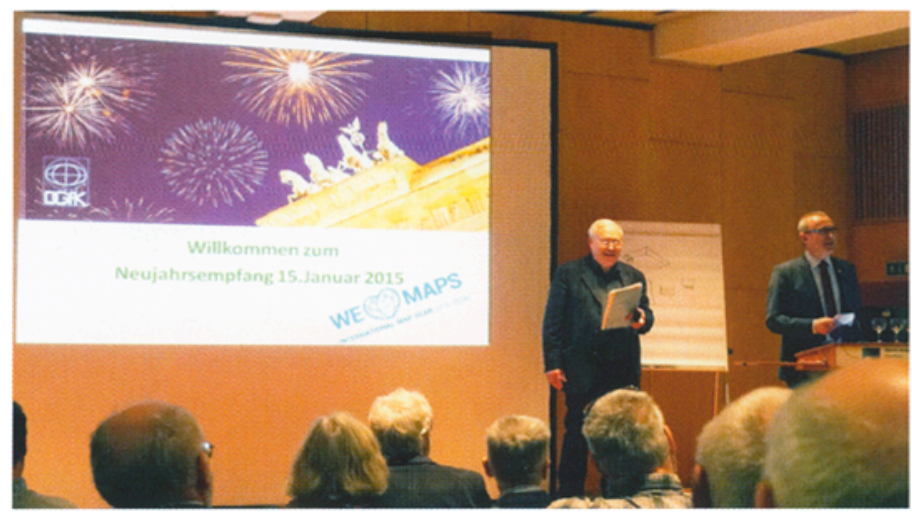

Der Leiter der Sektion Berlin-Brandenburg, H. Kremers und der Präsident der DGfK, M. Weisensee heißen die Besucher zum Neujahrsempfang willkommen.

Foto: Mark Vetter

der Universität Osnabrück gehalten. Seine erhellenden Ausführungen, die auf eine sehr angenehme Art heiter präsentiert wurden, standen unter dem Titel „Is spatial really special". Mit dem Vortrag teilte Herr Ehlers seine Gedanken „zur Rolle der Geo[infor] matik" mit dem Publikum. Der Abend endete mit zahlreichen Gesprächen in der Community am Buffet.

Mark Vetter, Karlsruhe

\section{Hochschul-}

\section{nachrichten}

\section{RIMMA}

(Risk Information

Management, Risk

Models and Applications)Workshop

Tagungsbericht

Am 17./18. November 2014 fand in Berlin der vierte einer Reihe von Workshops zum Themenbereich RISIKO statt. Organisiert wurde dieser internationale interdisziplinäre Workshop RIMMA (Risk Information Management, Risk Models and Applications) von CODATA-Germany, dem deutschen Nationalkomitee für das ICSU Committee on Data for Science and Technology in Kooperation mit der DGfK Kommission „Risiken, Katastrophen, Sicherheit". In bewährter Weise erfolgte der Austausch aktueller Erfahrungen und
Entwicklungen und es gab viel Raum für anregende Diskussionen über methodische Probleme in der Risikomodellierung, wobei der Schwerpunkt bei den Anforderungen an komplexen Informationssystemen für alle Phasen des Katastrophenzyklus lag.

Zu den Themen gehörten u.a.: Informationen für das operative Risiko-Management, Risiko-Datenbanken, Interoperabilität von Risiko informationen, Bedarf und Entwicklung von Standards, WebService-Strukturen, natürliche und technische Risiken von der lokalen bis zur internationalen Ebene, kartographische Fragen für Entscheidungsunterstützung beim Management von einzelnen Risiken oder bei Multi-(kaskadierenden) Risiken, die besondere Rolle von Kartographie und Geoinformation für Synergieeffekte durch raumbezogene Informations-Integration, Aufgaben für die Risikomodellierung bei kritischen Infrastrukturen (z. B. Fabriken, Eisenbahnen, Autobahnen, Pipelines, Seeverkehr usw.), operatives Katastrophenmanagement und Notfallvorsorge, Prävention, Alarm, Dokumentation, Archivierung und

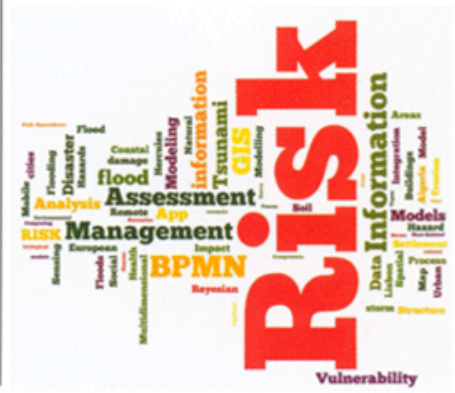

